\title{
City Emergency Command System Based on GIS
}

\author{
Yong Wang ${ }^{1, a}$, Jun Wang ${ }^{2, b}$ \\ ${ }^{1}$ School of Computer Science, Guangdong University of Technology, Guangzhou 510000, China; \\ ${ }^{2}$ School of Computer Science, Guangdong University of Technology, Guangzhou 510000, China. \\ a986715930@qq.com, b2412550795@qq.com
}

Keywords: GIS, gray multilevel analysis theory, city emergency command system

\begin{abstract}
For traditional urban scheduling system independent of all kinds of information, lack of information exchange and sharing between systems, scientific task evaluation and other issues, city emergency command system based on GIS was proposed. The whole system was developed under Windows platform , based on the architecture combining B/S mode and smart client. Business data and map data are stored in a relational database, using J2EE, GIS and GPS technology, combined with gray multi-level method to achieve the city's emergency command system with visual, scientific and other characteristics.
\end{abstract}

\section{Introduction}

With the accelerated pace of urban construction, man-made and natural emergencies happened frequently and more and more people are concerned about the city emergency command. Faced with the sudden disaster, the key of emergency rescue is how to access geospatial information about disaster area quickly, to assist the injured timely and evaluate result of the rescue scientific[1]. Geographic Information System (GIS) can manage all kinds of geographic information effectively and implement geospatial data's retrieval, analysis and presentation[2]. The construction of city emergency command system has some practical significance.

\section{System Structure}

Using 2D and 3D integration technology of GIS to display resources of the Dispatching process[3]. Using whiteboard to edit on the GIS map directly. The task's review is implemented by Task Management function. Using gray multilevel analysis theory to evaluate result of the rescue scientific. The system framework is shown in Figure 1:

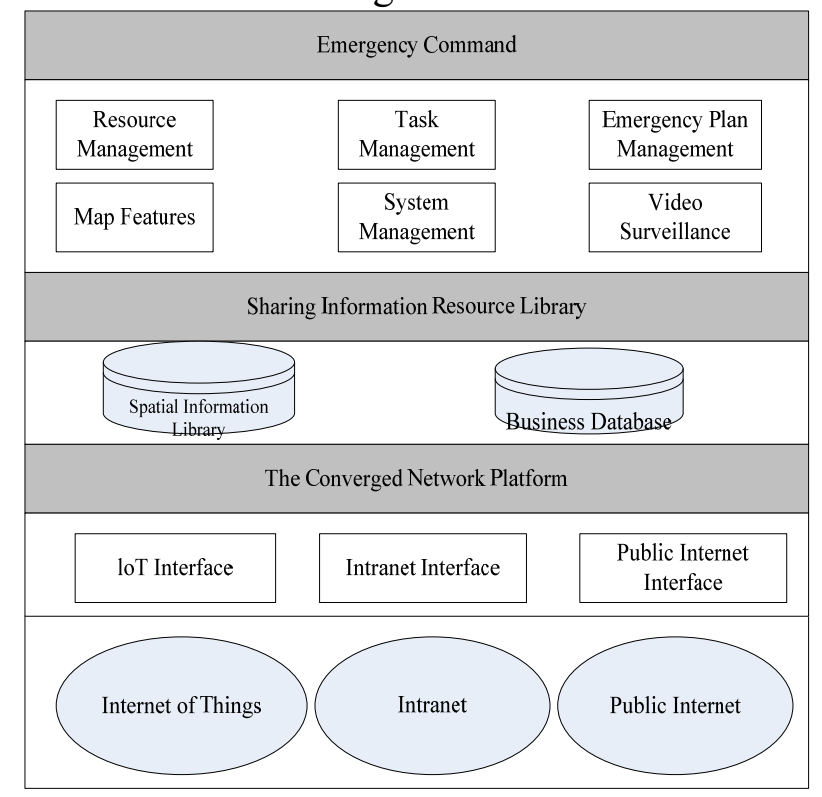

Figure 1 The System Structure 


\section{System function and database design}

\subsection{System fuction design.}

City Emergency Command System includes 6 phases:Recource Management, Task Management, Emergency Plan Management, Map Features, System Management, Video Surveillance. The main function are described in Figure 2:

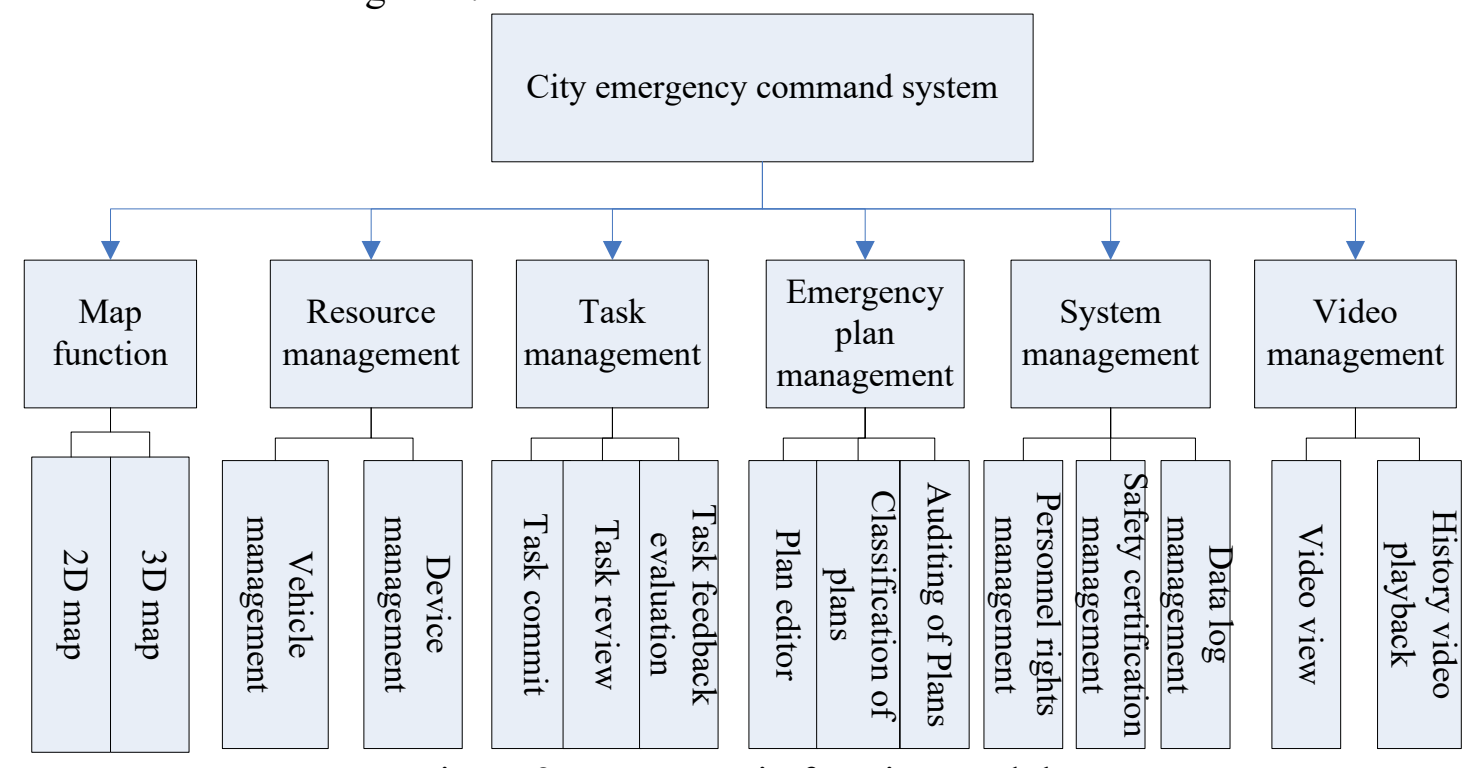

Figure 2 System main function module

1) Resource management. The system manage resources uniformly, including to add , modify, delete the basic parameters camera, vehicle and equipment.

2) Task Management. Staff can submit tasks to the system, Then the system will receive the task and the task will be reviewed. After the reviewing the task will be executed. the task will be evaluated after it has been completed.

3) Emergency Plan Management. For some common emergencies, the staff can edit the emergency plan in the system. In order to distinguish emergencies, classifying and arranging the plan.The system emergency plan approval function can ensure the plan more scientific.

4) Map Features. Using GIS technology to achieve the map of the two or three - dimensional display. The use of two or three dimensional map linkage can achieve the view of resources and emergency command in real time.

5) System Management. To manage the user's rights and ensure the security and consistency of the system.

6) Video Surveillance[4]. The view of video camera can be looked up in real time by clicking on the map of the video camera.

\subsection{Database design.}

Systems need to use the database to store all kinds of data. The database is the core of the information processing system. The system mainly include two kinds of data: geographic information data and business logic data. As the nature of the two kinds of data is not consistent, Storing data in spatial information database and service database.

1) Spatial information database. GIS can be used to achieve the spatial data and attribute data input and output functions. The spatial information database of the system is mainly to store the spatial information of urban geography. This system uses the SuperMap iObjects Java GIS application development platform which developmented by Beijing SuperMap software company.

2) Service database. Business logic data related to system resource management, user management and other functional modules is stored in the service database 


\section{Key technology}

\subsection{Task evaluation.}

After the review of the task, the relevant person will execute task according to the type of event. when the task is completed, the experts will evaluate the quality of the task. In order to avoid the experts' subjective judgment in the process of the evaluation of the qualitative method, the evaluation of the effect of the implementation of the plan must be combined with a certain quantitative calculation. The system has made a quantitative evaluation of the task completion by using the grey analytic hierarchy process. The general steps are as follows:

Step 1, to determine the emergency response evaluation index system, as shown in Table 1:

Table 1 Emergency Task Evaluation Index System

\begin{tabular}{|c|c|}
\hline First order index & Two level index \\
\hline \multirow{4}{*}{ Internal factor index $\left(\mathrm{U}_{1}\right)$} & Resource allocation $\left(\mathrm{U}_{11}\right)$ \\
\cline { 2 - 2 } & Personnel dispatch $\left(\mathrm{U}_{12}\right)$ \\
\cline { 2 - 2 } & Task complexity $\left(\mathrm{U}_{13}\right)$ \\
\cline { 2 - 2 } External factor index $\left(\mathrm{U}_{2}\right)$ & Clerical people factor $\left(\mathrm{U}_{14}\right)$ \\
\cline { 2 - 2 } & Media factor $\left(\mathrm{U}_{21}\right)$ \\
\cline { 2 - 2 } & Benefit factor $\left(\mathrm{U}_{22}\right)$ \\
\cline { 2 - 2 } & Masses factor $\left(\mathrm{U}_{23}\right)$ \\
\cline { 2 - 2 } & Government factor $\left(\mathrm{U}_{24}\right)$ \\
\hline
\end{tabular}

Step 2, to establish evaluation criteria. The evaluation index is divided into excellent, good, medium, poor and very poor according to the advantages and disadvantages.

Step 3, to determine the index weight of influencing factors of emergency task evaluation based on the AHP analytic hierarchy process. Analytical hierarchy process method, the qualitative and quantitative combination to solve the problem of decision making, will level factors pairwise comparison to determine the relative importance, so as to construct the judgment matrix and matrix characteristic value method for index weight.

Step 4, to construct evaluation sample matrix. Through a number of experts on the implementation of the plan two indicators scoring, and fill in the evaluation of the expert scoring table, which can be evaluated sample matrix.

Step 5, to determine the grey class evaluation. In order to objectively reflect the degree of belonging to a certain class, need to determine the evaluation of the grey class, that is, to determine the evaluation of the grade of the gray class, the number of gray ash and the number of whitening weight function.

Step 6, to calculate the grey evaluation coefficient.

Step 7, multi level comprehensive evaluation.

\subsection{Two,three-dimensional linkage.}

Two dimensional GIS has strong spatial analysis ability. And three-dimensional GIS display map about the spatial information more intuitive and clear. Two, three-dimensional linkage can give full play to the advantages of the two, to make up for each other's disadvantages. Two, three-dimensional linkage must ensure two synchronization: First, the operation of synchronization, Namely two, three-dimensional synchronous amplification, narrow, etc. This must correspond to the two dimensional coordinates of the map with the same name and the 3D geographic coordinates[5]. Second is data synchronization, Namely two-dimensional GIS and three-dimensional GIS reads the unified spatial database to ensure data synchronization.

\section{Summary}

This paper introduces the design and implementation of the urban emergency command system based on GIS. The system uses the grey analytic hierarchy process to carry on the scientific appraisal to the task quality .Using GIS two or three dimensional linkage technology to improve the analysis ability of spatial information and the visualization of monitoring resources. From many aspects to 
improve the rationality of urban emergency command, as far as possible to achieve visualization, scientific and intelligent, the construction of similar systems have some reference value.

\section{References}

[1]. Tolga Elbir. A GIS based decision support system for estimation, visualization and analysis of air pollution for large Turkish cities. Atmospheric Environment. Vol.38 (2004) No.27,p. 4509-4517

[2]. Andre Zerger,David Ingle Smith. Impediments to using GIS for real-time disaster decision support. Computers Environment and Urban Systems.Vol. 27 (2003) No.13,p.123-141.

[3]. Zhao Guimei. Application of integrated 2D and 3D GIS in the Ministry of Railways Dispatching System. Railway Signalling \& Communication Engineering.Vol.6 (2012) No.41 p.23-25

[4]. Liu Yanpeng, Yang Baozhu, Wang Yuansheng. Design of forest fire warning and command system based on browse/server architecture. Computer Engineering and Design. Vol.34(2013) No.1 p.360-365

[5]. Xiao Pengfeng,Feng Xuezhi,Huang Zhaoqiang,etal.Design of Urban Emergency System Using GIS and GPS Techniques.Remote Sensing Information.Vol 45 (2006) No.3 p.69-72 